SLAC-PUB-14346

January 2011

\title{
LESSONS LEARNED FROM A RECENT LASER ACCIDENT ${ }^{1}$
}

Paper \# 201

\author{
Michael Woods \\ SLAC National Accelerator Laboratory, \\ 2575 Sand Hill Rd., Menlo Park, CA 94025
}

\begin{abstract}
A graduate student received a laser eye injury from a femtosecond Ti:sapphire laser beam while adjusting a polarizing beam splitter optic. The direct causes for the accident included failure to follow safe alignment practices and failure to wear the required laser eyewear protection. Underlying root causes included inadequate on-the-job training and supervision, inadequate adherence to requirements, and inadequate appreciation for dimly visible beams outside the range of $400-700 \mathrm{~nm}$. This paper describes how the accident occurred, discusses causes and lessons learned, and describes corrective actions being taken.
\end{abstract}

\section{Description of Accident}

The laser activity involved aligning a polarizing beam splitter, which was used in an 800nm-wavelength beam path from a femtosecond Ti:sapphire (TiS) laser. The laser parameters were $808 \mathrm{~nm}$ wavelength, $100 \mathrm{fs}$ pulse width, $1 \mathrm{kHz}$ repetition rate and $100 \mathrm{~mW}$ beam power. With these beam parameters, laser eyewear protection (LEP) is required with an optical density (OD) greater than 4.0, which provides an attenuation factor of 10,000. The laser beam used came from a TiS laser system which had higher beam power capability than was used in the experiment - $2000 \mathrm{~mW}$ beam power and 40 fs pulse width, which would require the LEP to have an OD greater than 5.7.

The polarizing beam splitter, P1, was part of an optics configuration used for intensity control as shown in Figure 1. While adjusting the P1 optic, an unblocked reflected beam from it (dashed beam in Figure 1) hit the laser operator's eye. The operator was not wearing LEP at the time of the incident and sustained an injury, which resulted in a small blind spot in his peripheral vision. While the eye damage was relatively minor, the incident could have resulted in significant vision impairment.

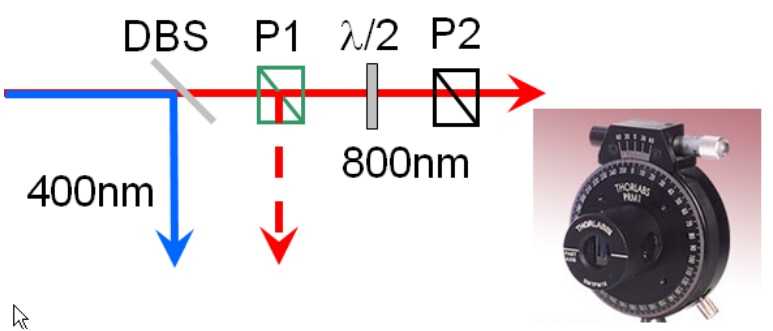

Figure 1: Optics configuration associated with the accident. DBS is a dichroic beam splitter; $\lambda / 2$ is a half waveplate; and P1 and P2 are polarizers, and P1 is mounted as shown in the photo at lower right.

The P1 polarizer optic has three components: a polarizer cube, which has an escape window for the reflection of one polarization component (the polarizer transmits one of the two linear polarization states and reflects the other one); a beam tube, which also has an escape window; and a rotation mount. The reflected beam from P1 is not used in the experiment and initially the beam tube and polarizer were correctly secured in the rotation mount so the beam tube would block the reflected beam.

The laser operator needed good extinction capability for controlling the laser intensity of the $800 \mathrm{~nm}$ beam, and wanted the polarizer optics to be normal to the beam path to help achieve this. To do this, the operator observed the back reflection from P1 on a fluorescent card while wearing the required LEP. However, the operator felt he could not get precise

1 Contributed to the 2011 International Laser Safety Conference, San Jose, CA; http://www.laserinstitute.org/conferences/ilsc/conference 
enough position information from the card because of a blooming effect due to saturation on the fluorescent card. The operator then decided to remove the LEP to allow viewing of the back-reflected beam on a white card. The operator also wanted to align the polarizer axis with a 0 -degree marking on its rotation mount -note that this task did not require the laser beam to be present. To get the rotation angle matched, he loosened the polarizer and the associated beam tube in the rotation mount to allow this adjustment. While doing so, the escape windows for the polarizer and beam tube became accidentally aligned and the unblocked reflected beam came out of the horizontal plane of the optical table into his left eye. Although the Standard Operating Procedure (SOP) document and the area warning signs specified that LEP was required for this activity, the laser operator was not wearing LEP at the time of the incident.

The alignment activity was for an experiment being performed by a small group of 2 graduate students and one post-doctorate (postdoc) researcher. The postdoc had a significant amount of laser experience, while the graduate students were relatively inexperienced. Their experiment was being conducted in a small research lab belonging to another research group, which was responsible for delivering a usable $800 \mathrm{~nm}$ beam for this experiment. One month after the experiment setup commenced, the postdoc left SLAC to take a research position elsewhere. The laser operator was working alone when the incident occurred, which was about two months after the initial experiment setup started.

\section{Direct Causes of Accident}

The direct causes of the accident were deficiencies in administrative alignment procedures used, failure to wear laser eyewear protection, and deficiencies in engineering controls. It took multiple failures and deficiencies for the accident to happen, indicating that the accident could have been prevented by implementing any one of a number of different controls.

\subsection{Deficiencies in Alignment Procedures.}

i. Aligning an optic normal to the beam. There are good techniques to do this while wearing LEP. These include:

- $\quad$ use an IR sensor card with a hole in it; incident beam goes through hole and one can view back-reflected beam on sensor card as polarizer pitch and yaw are adjusted
- $\quad$ cut an IR card so can have a straight edge of the card's sensitive part brought close to the beam (the more sensitive IR cards have a plastic coating and may not have sensitive material at the edge of the card)

- center an iris on the incident beam and close it until it just starts to clip the beam; then use a ccd camera or IR viewer to see the back-reflected beam from the optic being aligned on the iris

ii. Block or disable laser beams when not needed. The accident occurred when the worker was performing a procedure (orienting relative angle of the polarizer in its mount) that did not require the beam. Whenever the beam is not needed it should be blocked or disabled up beam of where work is being done.

iii. Do not perform unnecessary optics adjustments near accessible laser beams. The type of task being performed to align the relative angle of the polarizer axes with angle markings on the rotation mount should have been done in an optics preparation area away from there are accessible laser beams.

\subsection{Failure to Wear Laser Eyewear Protection}

The SOP and area warning signs indicated that LEP was required for this activity. The operator, however, made a bad decision to ignore this requirement.

\subsection{Deficiencies in Engineering Controls}

There were deficiencies in the optics hardware configuration to block the stray beam from the polarizer. Two configuration changes that would have prevented the accident are:

i. Use a beam tube with no escape window. For the experiment being done, the reflected beam from the polarizer was not needed.

ii. Use non-rotating mounts for the polarizing beam splitters, and mount them so the reflected beam is in the horizontal plane. Use half waveplates as needed to rotate the polarization vector of the laser beam.

\section{Root Causes for Accident}

The accident investigation and internal assessment of the lab's laser safety program needed to determine root causes for the accident, and go beyond the simple fact that the operator made a bad decision to perform laser work without the required LEP. Seven root causes were identified: 
i. Inadequate laser operator training. In particular, the on-the-job training (OJT) given for laser alignment tasks was inadequate.

ii. Inadequate supervision of laser operator by both the laser safety supervisor for the facility and by the worker's administrative supervisor that he reported to.

iii. Inadequate work planning: related to inadequate training and supervision mentioned above. There also was no prejob briefing or task review between the operator and a supervisor or experienced laser operator, which was needed given that the operator had limited laser alignment experience relevant for the tasks to be performed.

iv. Inadequate adherence to laboratory rules for laser alignment. This reflects the laser operator's decision to not wear the required $L E P$.

v. Inadequate intervention following (prior) laser eyewear safety violations by other laser operators in the facility. The injured laser operator had observed more senior laser operators remove required LEP to perform some tasks.

vi. Deceptive hazard of dimly visible beams outside 400-700nm wavelength range. An $800 \mathrm{~nm}$ beam is outside the normal visible range but is still dimly visible when viewed on a white card if it has high enough power. It does not appear hazardous, but if it can be seen the energy level is high enough to be a significant hazard.

vii. Inadequate appreciation for hazards associated with use of a polarizing beam splitter. Additional caution and expertise is needed when using an optic that can generate a beam out of the horizontal plane.

\section{Corrective Actions and Lessons Learned}

There was an externally-led investigation of the accident as well as internal assessments by the lab. These resulted in corrective actions for lab-wide programs at SLAC, specific corrective actions for the lab's laser safety program, and specific corrective actions for the facility where the accident occurred and for its laser personnel. Each Class 3B/4 laser facility at SLAC also conducted a self assessment. The self assessments were done by the facility's laser safety supervisor and an external reviewer, who was typically a laser safety supervisor for another facility. The self assessments reviewed the facility SOP document and the most recent facility inspection report by the SLAC Laser Safety Officer (LSO); a facility walkthrough was also conducted. The facility self-assessments were then reviewed by the LSO and line management for the facility. LSO and line management approval was required prior to resuming normal operations for each facility.

Corrective actions for lab-wide programs are directed at improved guidelines and requirements for OJT, supervision and facility operations. This paper is focused, though, only on corrective actions and lessons learned for the lab's laser safety program. These address the seven root causes that were identified in Section 3 and are now described. They were communicated to laser personnel via memos from the LSO and updates to classroom training classes (and will also be included in an update to the chapter on Laser Safety in the lab's Environment, Safety and Health Manual).

\subsection{Improvements to General Laser Safety Training}

i. Laser Accidents \& Lessons Learned classroom course. This is a new 90 -minute course taught by the LSO. It reviews the SLAC accident and other accidents that have occurred at Department of Energy (DOE) national laboratories [1]. Causes for these accidents and lessons learned from them are discussed. (Similar courses to this new SLAC course exist at Lawrence Berkeley National Lab and at Lawrence Livermore National Lab.)

ii. General Laser Safety web course. A new 3-hour course was implemented based on a relatively recent standardized DOE course for laser safety, which is being used at many of the DOE labs. This improvement was planned prior to the accident.

\subsection{Improvements to Hands-on and Site-specific Training}

i. Laser Alignment Practical course [2]. This is a new course that takes between 1-3 hours for students to complete, depending on their skill level and how many students are taking the course. The course is given by laser safety supervisors to a maximum of 3 students. Standardized practical training is given in core laser safety practices that are not site-specific. The course educates students on safe alignment techniques and common mistakes that are made. It also allows 
the laser safety supervisors to determine the skill level of new laser operators and determine how much supervision will be needed. (A similar course to the new SLAC course exists at Los Alamos National Lab.)

ii. Site-specific OJT. This training requires a good syllabus and must be documented for each laser operator. A corrective action was completed to create a template example for an OJT syllabus, with an associated OJT completion form. The syllabus has three components. The first is site orientation that instructs the new operator about operation modes for the facility, eyewear requirements for each operation mode, main components of the laser systems and safety features such as safety shutters and interlocks. Once site orientation is completed the new laser operator can begin work in the facility, but generally must have a more experienced laser operator present. The second part of the syllabus is for the laser operator to have demonstrated adequate alignment skills, and have adequate knowledge and competence to be able to work unsupervised in the facility. The operator may still have restrictions on what parts of the laser system they are approved to work on. The third part of the syllabus describes which specific laser systems the operator has received OJT for and approval to work on. It is also very important that OJT providers are adequately qualified to give OJT. Laser safety supervisors must train and approve laser operators who will give OJT; this is also documented in the OJT completion form.

\subsection{Improving Supervision}

SLAC has a Laser Supervisor Safety training course, which is a 2-hour classroom course given by the LSO. It is a relatively new course and was first given in August of 2009, not long before the laser accident occurred. At the time of the accident not all laser safety supervisors had completed the course, but all have since completed it. It has also been updated since the accident to reflect lessons learned and corrective actions from that.

Supervisor safety training emphasizes that supervisors are expected to:

- provide good site-specific OJT and supervision;

- communicate expectations for safe operations to laser operators;

- conduct pre-job briefings when appropriate, and encourage laser operators to request or conduct these as needed (see Section 4.4);
- $\quad$ ensure the laser facility is well managed; and

- perform frequent facility visits and interact with laser operators in their work.

Supervision responsibilities are more complex if there are shared supervision responsibilities, for example when the administrative supervisor a worker reports to is someone other than the laser safety supervisor. When there is shared or matrixed supervision, there needs to be clear communication between the different supervisors about supervision responsibilities. While the laser safety supervisor has direct responsibility for laser safety in their facility, the administrative supervisor still has a responsibility to ensure that their worker is adequately trained and supervised. Laser safety supervisors and administrative supervisors must together hold laser operators accountable for meeting laser safety requirements. One of the corrective actions implemented was to require administrative supervisors to sign approval for authorizing a laser operator to perform laser work in a given facility. Previously, approval was only required from the laser safety supervisor and the LSO.

\subsection{Improving Work Planning and Control}

SLAC has a comprehensive Work Planning and Control (WPC) program. Key WPC ingredients include the process and requirements for:

- evaluating and controlling hazards,

- authorizing and releasing work to be performed,

- conducting pre-job briefings, and

- stopping work if the work is outside the scope of what has been authorized and released, or if it could result in injury, or damage to the environment or equipment.

For laser work, the SOP document for a laser facility identifies the hazards and hazard controls. In some instances, a simpler Job Safety Analysis (JSA) document may also be used to extend the SOP description for specialized configurations and tasks.

Work authorization and release for laser operators is done with a laser operator approval form, which describes the training requirements and requires approval signatures from the facility's laser safety supervisor, the administrative supervisor for the operator, and from the LSO. Each Class 3B or Class 4 laser facility also has an operation approval from, which typically has a 1-year approval period. Facility operation approval requires an approved SOP or JSA document, a certification by the laser safety supervisor of the laser safety system (including safety interlock checks) and a facility inspection by the LSO. 
Guidance for pre-job briefings for laser work recommends that these be held in the following circumstances:

- new tasks,

- unfamiliar tasks or infrequently performed tasks,

- when there is a significant configuration change, or

- when a facility is returning to operation following an extended downtime.

Training emphasizes that pre-job briefings can be initiated by laser operators as well as by laser safety supervisors.

Concerning SLAC's stop work policy, all workers at the lab have both the responsibility and authority to stop work or to request stop work if the associated activity is outside the scope of what has been authorized and released, or if the worker perceives that the activity could lead to injury or damage to the environment or equipment. This is emphasized in the laser safety classroom training in addition to the training workers receive on this in the lab's general safety training courses.

\subsection{Improving Compliance with Safety Requirements}

To improve and facilitate compliance with laser safety requirements, there are several important aspects to address.

i. Safety training must review and stress the importance of complying with SOP (or JSA) requirements. This needs to include an emphasis on the importance of personal protective equipment (PPE), in particular LEP requirements for laser operators.

ii. Laser operators and supervisors have an important responsibility to identify and take action to fix inappropriate safety requirements and inadequate procedures. It is natural to encounter occasional problems when performing work that result from inadequate or incorrect procedures or inadequate equipment to perform a task. An important function for supervisors is to be able to effectively address such problems that arise and facilitate the process for doing this. It is unacceptable to find a work around that ignores a safety requirement and does not have proper work authorization and release.

iii. A significant challenge to supervisors is to make it easy to comply with laser safety requirements. WPC for laser work needs to address this in system configuration, available equipment (including laser eyewear) and procedures used.

iv. Supervisors, line management and senior workers need to establish and model a good safety culture. There has to be a consistent top-down and bottomup approach to safety, and it must be fully integrated with operations.

\subsection{Address Deceptive Hazard of Dimly Visible Laser Beams}

Figure 2 shows how the relative perceived brightness of a light source depends on its wavelength in the “visible region" from 400-700nm. A laser with 100 $\mathrm{mW}$ power at $700 \mathrm{~nm}$ will have a similar perceived brightness as one with $1 \mathrm{~mW}$ power at $550 \mathrm{~nm}$, when viewing a diffuse reflection on a white card without laser eyewear. The eye even has some sensitivity to wavelengths as long as $800 \mathrm{~nm}$.

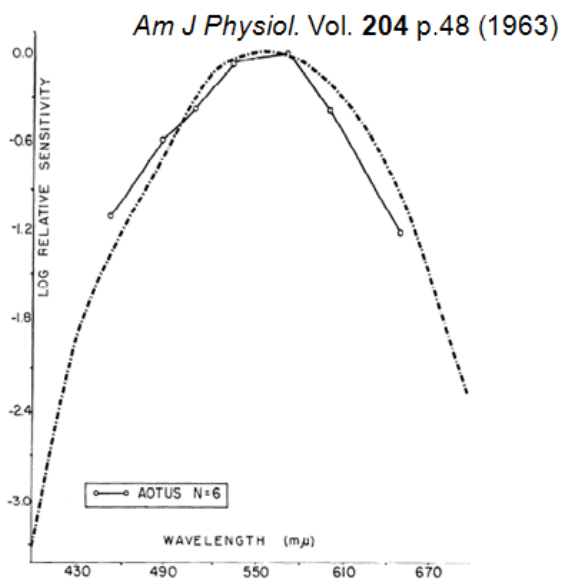

Figure 2: Relative sensitivity of the eye to wavelengths in the visible region, on a logarithmic scale.

Unfortunately some researchers and laser service personnel take advantage of dimly visible beams with wavelengths longer than 700nm to perform some tasks while not wearing LEP. They can become complacent about the associated hazard because the perceived hazard is less than what one observes with an alignment laser or a laser pointer. The laser power and pulse energies may also be small enough that the beam would not be felt if incident on the laser operator's hand, and often the diffuse reflection hazard can be below a hazardous level. The diffuse reflection hazard at 0.5-meter viewing distance from a white card is typically $10^{4}-10^{5}$ times less than the direct beam hazard. But while the diffuse reflection hazard may be ok, unintended stray beams at $(0.1-1) \%$ of the direct 
beam can be very hazardous. If LEP is removed for a task, one is often giving up OD5 or more in protection which is a huge amount of safety to give up.

Because of the prevalence of Ti:sapphire lasers operating at $700-800 \mathrm{~nm}$ wavelength, it is very important to directly address the hazard of dimly visible beams. If these beams were operating instead at $532 \mathrm{~nm}$ in the green, they would likely be scary enough that laser operators would want to wear the required $L E P$.

\subsection{Address Hazards Associated with Optics that Generate Out-of-plane Beams}

To the extent practical laser beams are always kept in the horizontal plane, and associated stray beams (ex. partial transmission in a dielectric mirror) are also in the horizontal plane. It is sometimes necessary, however, to use optics that generate out-of-plane beams. These optics include periscopes, polarizing beam splitters and diffraction gratings. Extra caution and special training is needed to be able to use these optics safely. The specialized training needs to be done in a practical course or in site-specific OJT. Laser operators need to have very good knowledge for how to safely use these optics, including how to set them up without hazards present, and how to implement appropriate beam blocks. They also need to be aware of common mistakes made that can lead to an injury.

\subsection{Take Appropriate Corrective and Disciplinary Actions for Safety Violations}

Supervisors have a responsibility to hold workers accountable for their job performance and for complying with safety requirements. Problems can arise in job performance or safety compliance, however, and how to address this is an important job function for supervisors. Often they are not given good guidance, though, on how to address safety violations or how to implement effective corrective actions. In most cases a verbal conversation should be adequate to address a safety issue, but if there is a pattern of ignoring safety requirements or if there is a severe safety violation then stronger corrective actions are needed.

There is a sequence of corrective actions that should be followed which progress in severity. These may start with counseling or an oral warning and then progress, if needed, to a written warning, suspension from performing an activity and, in some cases, even discharge from employment for gross misconduct or where attempts at corrective actions have failed.
Laser safety supervisors should not address safety violations by laser operators alone. The administrative supervisor and LSO should be consulted as well as other line management personnel, in particular if an oral warning is insufficient. It is also very important to understand why a safety violation occurred and what motivated the action, so that appropriate corrective actions can be developed. It may be necessary, for example, to acquire new equipment, modify a procedure, or conduct additional training as part of the corrective actions.

\subsection{Develop Performance Metrics for Laser Safety}

Course evaluation forms are useful to assess the quality and effectiveness of training courses and the instructors for these courses. It is also important, though more difficult, to assess and monitor the safety of laser operations. Site inspections by supervisors and the LSO, and discussions between them and laser personnel are important for this. Periodic surveys of laser personnel about the quality and effectiveness of OJT and laser safety supervision should also be undertaken (this is one corrective action that is not yet completed at SLAC). In general, performance metrics for laser safety is an area still requiring significant improvement at SLAC. Good performance metrics are important to help assess the risk for a laser accident, and also determine how best to mitigate the risk.

\section{Summary}

Multiple failures in administrative, engineering and PPE controls were the direct cause of a recent laser accident at SLAC. The accident analysis identified many root causes, including inadequate training and supervision, and inadequate appreciation for hazards of dimly visible beams outside the range of 400-700 nm. As a result, there were corrective actions for lab-wide programs and specific ones for the laser safety program. Lab-wide corrective actions include enhanced guidance and requirements for supervisors and for worker OJT. For the laser safety program, corrective actions include 2 new training courses for laser operators - a lessons learned classroom course taught by the LSO and a practical laser alignment course taught by laser safety supervisors. An improved site-specific OJT syllabus was developed and better guidance was given to laser safety supervisors on expectations for supervision, the need for pre-job briefings and how to address safety violations. 


\section{Acknowledgements}

Work supported by the U.S. Department of Energy under contract number DE-AC02-76SF00515.

\section{References}

[1] U.S. Department of Energy (2005) Special Operations Report: Laser Safety, http://www.hss.energy.gov/csa/analysis/reports/Laser Safety_Report.pdf.

[2] M. Woods and S. Edstrom (2011) Laser Safety: A Laser Alignment Practical Training Course, SLACPUB-14345; Paper \# 204 contributed to this conference.

\section{Meet the Author}

Michael Woods, CLSO, is the Laser Safety Officer at the SLAC National Accelerator Laboratory. He is an Engineering Physicist, with a B.Sc. in Engineering Physics from Queen's University in Kingston, Ontario, Canada and a Ph.D. in High Energy Physics from the University of Chicago. He has spent 15 years as a researcher in experimental particle physics and accelerator physics utilizing high power laser systems. He became SLAC LSO in 2008. 